\title{
LETTER
}

\section{Life history and spatial traits predict extinction risk due to climate change}

Richard G. Pearson ${ }^{1,2}$, Jessica C. Stanton ${ }^{3}$, Kevin T. Shoemaker ${ }^{3}$, Matthew E. Aiello-Lammens ${ }^{3}$, Peter

J. Ersts ${ }^{2}$, Ned Horning ${ }^{2}$, Damien A. Fordham ${ }^{4}$, Christopher J. Raxworthy ${ }^{2}$, Hae Yeong Ryu ${ }^{3}$, Jason

McNees $^{5}$, H. Reşit Akçakaya ${ }^{3 *}$

${ }^{1}$ Centre for Biodiversity and Environment Research, Department of Genetics, Evolution and

Environment, University College London, Gower Street, London, WC1E 6BT, UK.

${ }^{2}$ American Museum of Natural History, Central Park West at 79th Street, NY 10024, USA.

${ }^{3}$ Department of Ecology and Evolution, Stony Brook University, Stony Brook, NY 11794, USA.

${ }^{4}$ The Environment Institute and School of Earth \& Environmental Sciences, University of Adelaide, South Australia 5005, Australia.

${ }^{5}$ NatureServe, 1101 Wilson Blvd., 15th Floor, Arlington, VA 22209, USA.

*email: Resit.Akcakaya@stonybrook.edu

There is an urgent need to develop effective vulnerability assessments for evaluating the conservation status of species in a changing climate ${ }^{1}$. Several new assessment approaches have been proposed for evaluating the vulnerability of species to climate change $\mathrm{e}^{2-5}$, based on the expectation that established assessments such as the IUCN Red List ${ }^{6}$ need revising or superseding in light of the threat that climate change brings. However, although previous studies have identified ecological and life history attributes that characterize declining species or those listed as threatened ${ }^{7-9}$, no study to date has undertaken a quantitative analysis of the 
attributes that cause species to be at high risk of extinction specifically due to climate change. We developed a simulation approach based on generic life history types to show here that extinction risk due to climate change can be predicted using a mixture of spatial and demographic variables that can be measured in the present-day without the need for complex forecasting models. Most of the variables we found to be important for predicting extinction risk, including occupied area and population size, are already used in species conservation assessments, indicating that current systems may be better able to identify species vulnerable to climate change than previously thought. Therefore, although climate change brings many new conservation challenges, we find that it may not be fundamentally different from other threats in terms of assessing extinction risks.

Attempts to quantify the threat that climate change poses to species' survival commonly infer extinction risk from changes in the area of climatically suitable habitat (the bioclimate envelope) ${ }^{10,11}$, but this approach ignores important aspects of species' biology such as population dynamics, vital rates, and dispersal ${ }^{12-16}$, leading to high uncertainty ${ }^{1,17}$. To address this challenge, we coupled ecological niche models (ENMs) with demographic models ${ }^{13-15,18-20}$ and expanded this approach by developing a generic life history (GLH) method. The coupled modeling approach estimates extinction risk as the probability of abundance falling to zero by the year 2100 , rather than as the proportion of species committed to extinction due to contraction of bioclimate envelopes ${ }^{10}$ (Methods).

By matching ENMs for 36 amphibian and reptile species endemic to the US with corresponding GLH models (Supplementary Table S1), we estimate mean extinction risk by 2100 to be $28 \pm 7 \%$ under a high $\mathrm{CO}_{2}$ concentration Reference climate scenario ${ }^{21}$ and $23 \pm 7 \%$ under a Policy climate scenario that assumes substantive intervention ${ }^{22}$ (Methods). By contrast, extinction risk is estimated by the same models to be $<1 \%$ without climate change, showing that the methods are not biased toward predicting high risks. The contrast between predicted extinction risk with and without climate change suggests that climate change will cause a dramatic increase in extinction risk for these taxonomic 
groups over the coming century. Contrary to other studies ${ }^{23}$, the relatively small difference in extinction risk that we predict between Policy and Reference scenarios indicates that conservation actions that incorporate climate adaptation ${ }^{24}$ will be necessary to substantially reduce extinctions due to climate change regardless of whether mitigation measures that decrease $\mathrm{CO}_{2}$ emissions are implemented.

We next used sensitivity analysis to test how reliably extinction risk due to climate change can be predicted from current information on life history and spatial traits. Our goal was to assess whether commonly available variables can be used to effectively estimate extinction risk due to climate change without necessitating the application of complex forecasting models that are impractical to run for the majority of species. Based on the simulated period 2000-2010, we extracted 21 variables that could, in practice, be measured for conservation assessment purposes (Table 1). Application of machinelearning methods - Random Forests (RF) and Boosted Regression Trees (BRT) - revealed good ability to predict extinction risk due to climate change from these variables: $A U C=0.80-0.86$ based on cross validation partitioned by species so as to test using independent data (Methods).

The most important variables driving the predictions comprised a mixture of spatial and demographic factors (Fig. 1a). Occupied area was consistently the most important predictor, most likely because it provides a comprehensive measure of the breadth of climatic and habitat conditions under which a species can persist. Recent trends (calculated for the period 2000-2010), especially in occupied area, tend to be more important under the Policy scenario (Supplementary Fig. S1) than the Reference scenario (Fig. 1a; though recent trends were important under both scenarios when using BRT, Supplementary Figs. S2, S3). The finding that recent trends were less predictive of future trends under more severe climate change suggests that previously observed biological impacts of climate change ${ }^{25}$ will become less indicative of the future as climate change accelerates. Univariate plots reveal the general nature of the relationships between predictor variables and extinction risk due to climate change (Fig. 1b), although it is not always possible to 
[Page $218 \rightarrow$ ]

[Table 1: Predictor variables used to test whether extinction risk due to climate change can be predicted from present information on life history and spatial traits]

say whether extinction risk increases or decreases in response to a variable because of interactions between variables and non-linearity in these relationships.

Our analysis also revealed that species' vulnerability to climate change depends on interactions between life history traits and spatial characteristics (Fig. 2a). For example, the most important interaction under the Reference scenario was between a spatial variable (occupied area) and a demographic variable (generation length). The form of the interactions between variables is also informative. For example, although extinction risk due to climate change is highest when occupied area is small, the risk is lower when small occupied area is combined with long generation times (Fig. 2b). Relative to predictions from the full RF model (which allows for interactions), a fully additive approximation of the RF model (which assumes effects are mutually independent across parameter space for these two variables) miscalculated risk status by an average of $1.2 \%$ (RMSE) over the twodimensional parameter space (Fig. 2a). One measure of the importance of this interaction is that extinction risk due to climate change across the generation length spectrum varied only $1.4 \%$ for species with large occupied area, while the range was $8.2 \%$ for those with small occupied area (Fig 2b). The second most important interaction showed a similar form, wherein the effect of population size on extinction risk due to climate change was magnified for species with small occupied areas (Fig. 2c).

Some assessment systems already incorporate interactions between variables (for example, IUCN Red List criteria B and C include interactions between occupied area and recent trends; occupied area and fragmentation; and population size and recent trends ${ }^{6,26}$ ) but we found some interactions (such as between occupied area and generation length) that are not currently considered 
by vulnerability assessment systems. Further analyses of extinction risks with the approach used here will contribute to the development of guidelines to better account for climate change within conservation assessments ${ }^{12,26}$ and will inform the selection of essential biodiversity variables as part of a global observation system ${ }^{27}$.

The predicted large increases in probability of extinction under climate change forewarn that many species may be driven to extinction by climate change unless species-specific conservation actions are taken. Our results indicate that conservation actions should focus on species with small occupied area, small population

\section{[Page $219 \rightarrow$ ]}

[Figure 1: Predictors of extinction risk due to climate change by 2100]

size, spatially correlated dynamics, and declining occupied area, which are symptoms of endangerment used in the IUCN Red List criteria ${ }^{6,26}$ to identify species at risk of extinction. However, we caution that in evaluating conservation actions, species-specific coupled niche-demographic models should be used whenever data allow such models, especially in cases where the impact of climate change depends in part on species interactions ${ }^{28}$. We also emphasize that these results are based on amphibian and reptile species in the contiguous US and, although we present extensive sensitivity analyses, some effects of the taxonomic sampling are apparent. In particular, dispersal ability is not ranked among the most important predictors of vulnerability to climate change. This is because all species assessed in this analysis have similar and limited dispersal capacity, reducing our ability to identify this factor as important. Some potentially important factors are also excluded from the analyses. For example, we do not incorporate genetic adaptation that may facilitate survival over multiple generations but is not well understood in the context of rapid environmental change $\mathrm{e}^{29,30}$. Thus, we found lower extinction risk when generation length is long (Fig. 1b, 2b), but this is due to the effect of long lived species (e.g., box turtles) in our sample of life histories, which are less likely to go extinct 
within the timeframe of 100 years due to their longevity, and does not reflect the potential for short generation times to offer more potential for genetic adaptation (as well as faster demographic response to conservation measures) within the timeframe. Yet the approach we present offers great potential for application to additional taxonomic groups and in different regions, so that the results can be generalized and contribute to the development of effective measures to conserve biodiversity over the coming century.

\section{Methods}

We used an ensemble of five atmosphere-ocean general circulation models (GCMs) to generate an annual time series of climate anomalies to 2100 based on two strongly contrasting greenhouse gas emission scenarios: a Reference scenario with $\mathrm{CO}_{2}$ concentration of $750 \mathrm{ppm}(\mathrm{WRE} 750)^{21}$ and a Policy scenario with $\mathrm{CO}_{2}$ stabilization at $450 \mathrm{ppm}\left(\right.$ MiniCAM LEV1) ${ }^{22}$. Climate anomalies were downscaled to an ecologically relevant spatial resolution ( 800m $\times 800 \mathrm{~m})$ and nineteen bioclimate variables were generated, from which seven variables were selected based on reasoning as to the physiological and life-history requirements of the study species and analysis of correlations between variables.

We then combined the seven climate variables with other environmental variables (including land cover, hydrography and land surface form) and species' occurrence records to generate annual maps of suitable habitat using ecological niche models (ENMs). Occurrence data were obtained from NatureServe and we used the maximum entropy ENM method (Maxent). Maxent regularization was set for each species individually so as to avoid over-fitting, and the most relevant land cover, hydrography, and land surface form variables were selected for each species to avoid fitting models with an unnecessarily large number of variables (Supplementary Table S2). The extent of the study region for ENM calibration was selected for each species based on occupied ecoregions, and for each 
species the final ENM was a consensus from 50 replicates so as to account for uncertainty in the species' occurrence data.

The dynamic spatial structures generated by ENMs were then combined with generic life history (GLH) models, which incorporate demographic structure, density dependence and stochasticity, to create metapopulation models (i.e., spatially structured models of multiple populations, with partially correlated dynamics, that may exchange individuals through dispersal). We constructed age- and stage-structured, density-dependent, stochastic models for 6 life history types: small salamanders, large salamanders, turtles, tortoises, snakes, and lizards. From these GLH models we created 9,720 population models (3,240 for each climate scenario) by sampling a standard set of life history parameters between upper and lower bounds. Using a generic approach for modeling demographic processes prevents us from making species-specific projections or ranking these 36 species in terms their vulnerability to climate change. But it also avoids the need to obtain species-specific parameters, which are rarely known, and extends our scope of inference beyond a limited set of extant species to all possible trait combinations that may increase risk of extinction due to climate change. To estimate the risk of extinction we ran each of the 9,720 population models for 1,000 replicates, and each replicate with annual time steps to 2100 . We also estimated extinction risk without climate change and we did not model non-climatic threats, such as habitat destruction or exploitation, enabling us to isolate the degree to which climate change increases extinction risk.

We then used machine-learning methods, RF and BRT, to identify important variables and interactions for predicting extinction risk due to climate change. We assessed RF and BRT model predictive ability using leave-one-out cross validation in which each of the 36 species was treated in turn as an

[Page $220 \rightarrow$ ]

[Figure 2: Role of interactions between variables in predicting extinction risk due to climate change] 
independent validation data set. By using species as a data partition instead of random sampling methods (e.g., standard 10-fold cross-validation), we were able to challenge the modeling algorithm against truly independent data. Thus, the predictive performance metrics we calculated (AUC=0.86 for RF under Reference scenario; for other results, see Supplementary Methods) could be expected to hold for predictions of climate-related risk to additional North American species not included in this study. For RF, importance of each predictor variable was determined by computing the prediction error of each tree for the out-of-bag sample (i.e., set of observations set aside for validation and not used in constructing the trees) and assessing the degree to which out-of-bag prediction error increases when the values of that predictor variable are randomly shuffled. Univariate relationships between variables and extinction risk due to climate change (Fig. 1b) were derived post-hoc by predicting across the parameter space for each variable while holding all other variables constant at mean values. Two-way interaction strengths were computed post hoc following three steps: 1. For each variable pair, predictions were made across the full two dimensional slice of parameter space (holding all other predictor variables constant at mean values); 2. Predictions from step 1 were modeled assuming additivity (but the relationship is not constrained to be linear and could take any shape); and 3 . The root mean squared residual error under the additive model from step 2 (multiplied by 100 to convert to percent risk) was calculated as an index of the strength of interaction. For BRT, importance of each predictor variable was computed as the total reduction in deviance associated with that variable for the full model.

Supplementary Fig. S4 presents a flowchart detailing processing steps and data inputs/outputs. Further explanation and justification of data and methods is provided in Supplementary Methods.

\section{References}

1. Dawson, T. P., Jackson, S. T., House, J. I., Prentice, I. C. \& Mace, G. M. Beyond predictions: Biodiversity conservation in a changing climate. Science 332, 53-58 (2011). 
2. Foden, W. B. et al. Identifying the World's Most Climate Change Vulnerable Species: A Systematic Trait-Based Assessment of all Birds, Amphibians and Corals. PLoS ONE 8, e65427 (2013).

3. Thomas, C. D. et al. A framework for assessing threats and benefits to species responding to climate change. Methods Ecol. Evol. 2, 125-142 (2011).

4. Young, B. E. et al. in Wildlife Conservation in a Changing Climate 129-152 (University of Chicago Press, 2012).

5. Gillson, L., Dawson, T. P., Jack, S. \& McGeoch, M. A. Accommodating climate change contingencies in conservation strategy. Trends Ecol. Evol. 28, 135-142 (2013).

6. Mace, G. M. et al. Quantification of extinction risk: IUCN's system for classifying threatened species. Conserv. Biol. 22, 1424-1442 (2008).

7. Collen, B. et al. Predicting how populations decline to extinction. Phil. Trans. R. Soc. B 366, 25772586 (2011).

8. Purvis, A., Gittleman, J. L., Cowlishaw, G. \& Mace, G. M. Predicting extinction risk in declining species. Proc. R. Soc. Lond. B 267, 1947-1952 (2000).

9. Cardillo, M. et al. Multiple causes of high extinction risk in large mammal species. Science 309, 1239-1241 (2005).

10. Thomas, C. D. et al. Extinction risk from climate change. Nature 427, 145-148 (2004).

11. Thuiller, W., Lavorel, S., Araújo, M. B., Sykes, M. T. \& Prentice, I. C. Climate change threats to plant diversity in Europe. PNAS 102, 8245-8250 (2005).

12. Akçakaya, H. R., Butchart, S. H. M., Mace, G. M., Stuart, S. N. \& Hilton-Taylor, C. Use and misuse of the IUCN Red List Criteria in projecting climate change impacts on biodiversity. Glob. Change Biol. 12, 2037-2043 (2006).

13. Fordham, D. A. et al. Plant extinction risk under climate change: are forecast range shifts alone a good indicator of species vulnerability to global warming? Glob. Change Biol. 18, 1357-1371 (2012). 
14. Fordham, D. A., Akçakaya, H. R., Araujo, M. B., Keith, D. A. \& Brook, B. W. Tools for integrating range change, extinction risk and climate change information into conservation management. Ecography (2013). doi:10.1111/j.1600-0587.2013.00147.x

15. Keith, D. A. et al. Predicting Extinction Risks Under Climate Change: Coupling Stochastic Population Models with Dynamic Bioclimatic Habitat Models. Biol. Lett. 4, 560-563 (2008).

16. Buckley, L. B. et al. Can mechanism inform species' distribution models? Ecol. Lett. 13, 10411054 (2010).

17. Pereira, H. M. et al. Scenarios for global biodiversity in the 21st century. Science 330, 1496-1501 (2010).

18. Schurr, F. M. et al. How to understand species' niches and range dynamics: a demographic research agenda for biogeography. J. Biogeogr. 39, 2146-2162 (2012).

19. Buckley, L. B. Linking Traits to Energetics and Population Dynamics to Predict Lizard Ranges in Changing Environments. Am. Nat. 171, E1-E19 (2008).

20. Dullinger, S. et al. Extinction debt of high-mountain plants under twenty-first-century climate change. Nature Clim. Change 2, 619-622 (2012).

21. Wigley, T. M. L., Richels, R. \& Edmonds, J. A. Economic and environmental choices in the stabilization of atmospheric CO2 concentrations. Nature 379, 240-243 (1996).

22. Wigley, T. M. L. et al. Uncertainties in climate stabilization. Climatic Change 97, 85-121 (2009).

23. Warren, R. et al. Quantifying the benefit of early climate change mitigation in avoiding biodiversity loss. Nature Clim. Change 3, 678-682 (2013).

24. Mawdsley, J. R., O'malley, R. \& Ojima, D. S. A review of climate-change adaptation strategies for wildlife management and biodiversity conservation. Conserv. Biol. 23, 1080-1089 (2009).

25. Rosenzweig, C. et al. Attributing physical and biological impacts to anthropogenic climate change. Nature 453, 353-357 (2008).

26. IUCN Standards and Petitions Subcommittee. Guidelines for using the IUCN Red List categories and criteria (Version 10). (2013). at <www.iucnredlist.org/documents/RedListGuidelines.pdf>

27. Pereira, H. M. et al. Essential Biodiversity Variables. Science 339, 277-278 (2013). 
28. Fordham, D. A. et al. Adapted conservation measures are required to save the Iberian lynx in a changing climate. Nature Clim. Change 3, 899-903 (2013).

29. Hoffmann, A. A. \& Sgrò, C. M. Climate change and evolutionary adaptation. Nature 470, 479-485 (2011).

30. Chevin, L.-M., Lande, R. \& Mace, G. M. Adaptation, plasticity, and extinction in a changing environment: Towards a predictive theory. PLoS Biol 8, e1000357 (2010).

Acknowledgements Funding was provided by NASA (Biodiversity Program grant NNX09AK19G). The Australian Research Council supported D.A.F. (grants LP0989420, DP1096427and FS110200051). J. Palmer, S. Phillips, J. Ray, A. Sonneborn helped with analyses, G. Mace provided comments on a draft manuscript, and B. Young, NatureServe and its Natural Heritage member programs provided data.

Author Contributions R.G.P. and H.R.A. designed the study, analysed data, and led writing the manuscript; R.G.P. ran ENM models; H.R.A. developed GLH models; J.C.S. selected variables for ENM and linked ENM and GLH models; J.C.S. and H.Y.R. collated demographic data and ran metapopulation simulations; K.T.S. led BRT and RF analyses; M.E.A.-L. sampled models from GLH and extracted simulation results; P.J.E. and N.H. developed ENM input data; D.A.F. led climate analyses; C.J.R. helped with species selection, variable selection and demographic data; J.M. collated species data; all authors discussed results and contributed to writing the manuscript.

Author Information Climate data are available through the NASA Center for Climate Simulation (www.nccs.nasa.gov; dx.doi.org/10.7917/D7WD3XH5). Correspondence and requests for materials should be addressed to H.R.A.

Competing financial interests The authors declare no competing financial interests. 


\section{Figure Legends}

Figure 1. Predictors of extinction risk due to climate change by 2100 . Results are for Random Forest models under the Reference climate change scenario. (a) Importance of each predictor variable, computed as the relative loss in predictive performance after shuffling (i.e., randomly reordering) the values of that predictor variable (Supplementary Methods). Demographic and spatial variables were estimated for the year 2000; recent trend variables were estimated from the simulated period 2000 to 2010. (b) Univariate relationships between extinction risk due to climate change and the four most important predictor variables. $Y$-axes are scaled so that 0.0 is the mean value of the response.

Figure 2. Role of interactions between variables in predicting extinction risk due to climate change. (a) Strength of two-way interactions between determinants of extinction risk due to climate change (Reference scenario). The six strongest two-way interactions are shown. (b) and (c) Threedimensional visualization of the first and second ranked interactions, respectively. Results are based on Random Forest models. 
Table 1. Predictor variables used to test whether extinction risk due to climate change can be predicted from current information on life history and spatial traits.

\begin{tabular}{|c|c|}
\hline Variable & Explanation \\
\hline Population size & Number of individuals in all populations, log-transformed \\
\hline Generation length & $\begin{array}{l}\text { Generation length in years; measures the rate of demographic turnover or speed } \\
\text { of life history }\end{array}$ \\
\hline Growth rate & Average rate of population growth at low population sizes (Rmax) \\
\hline Vital rate variability & $\begin{array}{l}\text { Coefficient of variation of vital rates (survival rates and fecundities for each age } \\
\text { class or stage), averaged over all non-zero rates; measures the tendency of the } \\
\text { population to fluctuate in response to stochastic environmental change }\end{array}$ \\
\hline Spatial correlation & $\begin{array}{l}\text { Degree to which environmental variability is correlated across the landscape; } \\
\text { coefficient of correlation at the average distance among occupied populations }\end{array}$ \\
\hline $\begin{array}{l}\text { Number of } \\
\text { subpopulations }\end{array}$ & Number of extant subpopulations (i.e., patches with at least 1 individual) \\
\hline Occupied area & $\begin{array}{l}\text { Total area of all occupied patches (log-transformed); corresponds to area of } \\
\text { occupancy (AOO) used in IUCN Red List criteria }{ }^{26} \text {, measured at }<1 \mathrm{~km} \text { scale. }\end{array}$ \\
\hline Fractal dimension & $\begin{array}{l}\text { A measure of habitat fragmentation, calculated as } 2 \cdot \ln (0.25 \cdot E) / \ln (A) \text {, where } E \text { is } \\
\text { the total edge length of all patches (in units of cell length), and } A \text { is the total area of } \\
\text { all patches (in number of cells) }\end{array}$ \\
\hline $\begin{array}{l}\text { Largest patch } \\
\text { fraction }\end{array}$ & Area of the largest patch as a fraction of total metapopulation area \\
\hline Connectivity & $\begin{array}{l}\text { A measure of dispersal between neighboring populations, calculated as the } \\
\text { dispersal rate at the distance to nearest neighbor (averaged over all extant } \\
\text { subpopulations) using the dispersal function }\end{array}$ \\
\hline Fragmentation & $\begin{array}{l}\text { Degree of demographic fragmentation, measured as the proportion of all } \\
\text { individuals that are in small and isolated populations. Values }>0.5 \text { correspond to } \\
\text { "severely fragmented" as defined in ref. }{ }^{26}\end{array}$ \\
\hline Dispersal ability & $\begin{array}{l}\text { Intrinsic dispersal ability: Distance above which only } 1 \% \text { of individuals are likely to } \\
\text { disperse; likely maximum dispersal distance for } 99 \% \text { of individuals in a given year. }\end{array}$ \\
\hline $\begin{array}{l}\text { Niche breadth: } \\
\text { temperature }\end{array}$ & Range of average annual temperatures in the current range \\
\hline $\begin{array}{l}\text { Niche breadth: } \\
\text { precipitation }\end{array}$ & Range of average annual precipitation in the current range \\
\hline $\begin{array}{l}\text { Trend in occupied } \\
\text { area }\end{array}$ & Ten-year trend (2000-2010) in occupied area \\
\hline $\begin{array}{l}\text { Trend in population } \\
\text { size }\end{array}$ & Ten-year trend (2000-2010) in total abundance \\
\hline $\begin{array}{l}\text { Trend in number of } \\
\text { subpopulations }\end{array}$ & Ten-year trend (2000-2010) in the number of subpopulations \\
\hline $\begin{array}{l}\text { Trend in fractal } \\
\text { dimension }\end{array}$ & Ten-year trend (2000-2010) in fractal dimension \\
\hline $\begin{array}{l}\text { Trend in } \\
\text { connectivity }\end{array}$ & Ten-year trend (2000-2010) in connectivity \\
\hline $\begin{array}{l}\text { Trend in } \\
\text { fragmentation }\end{array}$ & Ten-year trend (2000-2010) in fragmentation index \\
\hline $\begin{array}{l}\text { Trend in largest } \\
\text { patch fraction }\end{array}$ & $\begin{array}{l}\text { Ten-year trend (2000-2010) in the proportion of total metapopulation area that is in } \\
\text { the largest patch }\end{array}$ \\
\hline
\end{tabular}

